\title{
Higher-order anisotropies in the Blast-Wave Model - disentangling flow and density field anisotropies
}

\author{
Jakub Cimerman ${ }^{1,2}$, Boris Tomášik ${ }^{1,3}$, Máté Csanád ${ }^{4}$, and Sándor Lökös ${ }^{4}$ \\ 1 Czech Technical University in Prague, FNSPE, Břehová 7, 11519 Prague 1, Czech Republic \\ 2 Comenius University, FMPI, Mlynská Dolina F1, 84248 Bratislava, Slovakia \\ 3 Univerzita Mateja Bela, FPV, Tajovského 40, 97401 Banská Bystrica, Slovakia \\ 4 Eötvös Loránd University, Pázmány P. s. 1/a, H-1117 Budapest, Hungary
}

\begin{abstract}
We formulate a generalisation of the blast-wave model which is suitable for the description of higher order azimuthal anisotropies of the hadron production. The model includes anisotropy in the density profile as well as an anisotropy in the transverse expansion velocity field. We then study how these two kinds of anisotropies influence the single-particle distributions and the correlation radii of two-particle correlation functions. Particularly we focus on the third-order anisotropy and consideration is given averaging over different orientations of the event plane.
\end{abstract}

PACS. 25.75.Dw , 25.75.Gz, 25.75.Ld

\section{Introduction}

The hot matter excited in ultrarelativistic heavy-ion collisions at colliders like the LHC or RHIC exhibits a sizeable anisotropy in particle production perpendicularly to the beam direction $1,2,2,3,4,5$. The azimuthal anisotropy of hadron momentum distributions, measured in terms of Fourier coefficients, is caused by the anisotropy of the fireball at freeze-out in spatial density and expansion pattern. That, in turn, results from its evolution which starts from anisotropic initial conditions [6,7,8,9,10, and may further receive anisotropic excitations on the way [11,12,13, 14. The evolution depends on the Equation of State and the transport coefficients [15, 16, 17]. Thus by measuring the final state anisotropies one gets an access to the intrinsic properties of the matter [18. Note that the anisotropies are unique in each event and a large fraction of them, especially higher-order anisotropies, are averaged out if measured in a sample consisting of a large number of events.

Hadrons are emitted at the moment of freeze-out and this is when their distributions are formed. Hence, the direct information that they are carrying is about the state of the fireball at this very moment. The present paper deals with this relation. It is then the task for hydrodynamic and/or transport simulations to conclude about the preceding evolution of the fireball.

There are two kinds of anisotropies of the fireball at the moment of freeze-out, that may cause an anisotropy of the hadron distribution 19,20,21,22. Firstly, if the transverse expansion velocity in some directions is higher than in the others, the stronger blueshift of the momentum spectra in those directions will cause momentum anisotropy. Secondly, alone an expanding and spatially anisotropic fire- ball may also produce an anisotropy of the momentum distribution. Unfortunately, the two mechanisms cannot be distinguished by mere measurement of the momentum anisotropies.

For the second-order anisotropies it has been shown that the solution is offered by the azimuthal dependence of the femtoscopic correlation radii, which are more sensitive to spatial anisotropy. Detailed studies with the help of blast-wave 19 and Buda-Lund 20 models have been performed. After PHENIX has published the azimuthal dependence of the correlation radii 23 with respect to the third-order event plane, the problem has been reconsidered at that order in 21. It has been demonstrated in framework of a toy model that at RHIC the spatial anisotropy is the driving feature which determines the phase of the oscillation of the correlation radii. This is in agreement with the second-order results 19,20. Today, higher experimental statistics allows for more detailed investigations and the data on third-order azimuthal dependence of correlation radii call for more detailed theoretical studies [25, 26, 27]. The third-order anisotropy together with azimuthal dependence of the correlation radii have been investigated by some of us in detail in framework of the Buda-Lund model recently 22 . The dependence of the oscillation amplitudes on parameters of the model which gauge the anisotropies in space and flow has been calculated in great detail up to 6 th order.

The present paper is analogical to 22 . We reconsider the problem in framework of the blast-wave model. This is perhaps the most commonly used model for the analysis of soft hadron data in high energy heavy-ion physics. Therefore, we first systematically extend it for anisotropies of 
higher order, although we later use it only up to the third order. By doing this we actually modify the formulation of the model as proposed in 32, since that one was adequate only up to second order. Note that our extension also slightly differs from the one proposed in 24, since want to allow for varying transverse size of the fireball.

Then, with the generalised blast-wave model we investigate in quite detail how the oscillation amplitudes of the correlation radii depend on parameters which measure the flow and the shape anisotropy. On top of the schematic picture obtained in 21 we add the details by providing parameter maps, i.e. contour plots of the dependence of oscillation amplitudes on both flow and space anisotropy, similarly as was done for the Buda-Lund model in 22. Such maps should allow, at least in principle, to infer both values for flow and space anisotropy from the measured data. We also go beyond the toy-model study of 21] by including both the second and the third order anisotropies into the calculation and integrating over the order not actually being investigated, analogically to real experiment.

In the next Section we introduce and explain the extension of the blast-wave model used in this study. Then, Section 3 is devoted to calculations of the anisotropies of single-particle distributions. Oscillations of correlation radii are investigated in Section 4 . We demonstrate our results with the help of qualitative data analysis in Section 5. All results are summarised in the concluding Section. Some technical details are explained in the Appendices.

\section{The extended blast-wave model}

Particle production is described with the help of the emission function $S(x, p)$, which is the Wigner function, i.e. the phase-space density of hadrons that are being emitted from the fireball. In the blast-wave model [28, 29, 30, 31, 32 it is parametrised as

$$
\begin{aligned}
& S(x, p) d^{4} x=\frac{g}{(2 \pi)^{3}} m_{t} \cosh \left(Y-\eta_{s}\right) \\
& r d r d \theta \tau d \eta \frac{d \tau}{\sqrt{2 \pi} \Delta \tau} \exp \left(-\frac{\left(\tau-\tau_{0}\right)^{2}}{2 \Delta \tau^{2}}\right) \\
& \Theta(r-R(\theta)) \exp \left(-\frac{p^{\mu} u_{\mu}}{T}\right) .
\end{aligned}
$$

Here, $g$ is the spin degeneracy factor, $T$ is the local temperature, $u^{\mu}$ is the expansion velocity field, and $\Theta(x)$ is the Heaviside step function. We parametrise the momentum of a particle with the help of transverse momentum $p_{t}$, transverse mass $m_{t}$, rapidity $Y$ and the azimuthal angle $\phi$ as

$$
p^{\mu}=\left(m_{t} \cosh Y, p_{t} \cos \phi, p_{t} \sin \phi, m_{t} \sinh Y\right) .
$$

In this paper we shall denote the rapidity with capital letter in order to distinguish it from the spatial coordinate.

As spatial coordinates we use the radial coordinate $r$ and the azimuthal angle $\theta$, as well as the space-time rapidity $\eta_{s}=\frac{1}{2} \ln ((t+z) /(t-z))$ and longitudinal proper time $\tau=\sqrt{t^{2}-z^{2}}$. Then

$$
x^{\mu}=\left(\tau \cosh \eta_{s}, r \cos \theta, r \sin \theta, \tau \sinh \eta_{s}\right) .
$$

Furthermore, $R(\theta)$ is the transverse size of the fireball, depending on the azimuthal angle $\theta$. The spatial anisotropy of the model is specified by the particular prescription for $R(\theta)$. The transverse size is then parametrised as

$$
R(\theta)=R_{0}\left(1-\sum_{n=2}^{\infty} a_{n} \cos \left(n\left(\theta-\theta_{n}\right)\right)\right)
$$

where the amplitudes $a_{n}$ and the phases $\theta_{n}$ are model parameters. Note that $\theta_{n}$ 's denote the orientations of the so-called $n$-th order event planes. Note that in the series we have skipped the first-order term which leads to mere shift of the shape. Also note that the amplitudes for the oscillation are parametrised in an unusual way, with the help of $\left(-a_{n}\right)$. In this way, the resulting $v_{n}$ is to first order proportional to $a_{n}$, as will be seen later.

Note that in 19 a different parametrisation for the elliptic shape of the fireball was used, with radii $R_{x}$ and $R_{y}$ along the two axes of the ellipse

$$
R_{x}=a R^{\prime}, \quad R_{y}=\frac{R^{\prime}}{a}
$$

with $R^{\prime}$ and $a$ being parameters of the model. The advantage of that parametrisation is that when $a$ is being tuned, the volume stays constant and proportional to $R^{2}$. Nevertheless, such a prescription cannot be naturally generalised to higher orders. Therefore, we will now use Eq. (4). Some comments on the relation of the two parametrisations can be found in Appendix A.

It is convenient for further calculation to define a dimensionless transverse coordinate

$$
\bar{r}=\frac{r}{R(\theta)}
$$

Particle production in our model occurs for $\bar{r}$ in the range $[0,1]$.

Expansion is described by the velocity field $u^{\mu}$. Velocity includes longitudinal as well as transverse component

$$
\begin{aligned}
u^{\mu}=\left(\cosh \eta_{s} \cosh \rho, \sinh \rho \cos \theta_{b},\right. \\
\left.\quad \sinh \rho \sin \theta_{b}, \sinh \eta_{s} \cosh \rho\right)
\end{aligned}
$$

where

$$
\theta_{b}=\theta_{b}(r, \theta)
$$

is the angle of the transverse vector of the velocity and will be specified below. Furthermore

$$
\rho=\rho\left(\bar{r}, \theta_{b}\right)
$$

is the rapidity connected with the transverse velocity, so that the transverse velocity at midrapidity is $v_{t}=\tanh \rho$.

The canonical (azimuthally symmetric) blast-wave model is recovered if $R(\theta)$ and $\rho(\bar{r}, \theta)$ do not depend on the angle and $\theta_{b}=\theta$. Here we construct the extension to arbitrary order of anisotropy. 
In a fireball without azimuthal symmetry we must specify the direction of the transverse expansion velocity. In [19, two models were investigated which differed in the choice of that direction. Note, however, that only secondorder anisotropy was studied there. It turned out that femtoscopic data [25] agreed with the choice in which the transverse velocity was always perpendicular to the surface of the fireball. We adopt this choice also here. Transverse velocity will be locally perpendicular to surfaces with constant $\bar{r}$. Note that this is the natural direction of transverse pressure gradient and thus the acceleration. Hence, we actually identify the direction of the velocity with that of acceleration. Such a choice is expected to be valid if the fireball decouples fast. from

The azimuthal angle of the velocity $\theta_{b}$ is then obtained

$$
\tan \left(\theta_{b}-\frac{\pi}{2}\right)=\frac{d y}{d x}=\frac{\frac{d y}{d \theta}}{\frac{d x}{d \theta}}
$$

where the derivative is taken along a surface with constant $\bar{r}$. The solution is straightforward and we summarise it together with the final result for $\theta_{b}$ in Appendix B

Finally, let us define the magnitude of the transverse velocity, which is parametrised with the help of transverse rapidity

$$
\rho\left(\bar{r}, \theta_{b}\right)=\bar{r} \rho_{0}\left(1+\sum_{n=2}^{\infty} 2 \rho_{n} \cos \left(n\left(\theta_{b}-\theta_{n}\right)\right)\right) .
$$

Overall transverse flow is tuned with the help of $\rho_{0}$ and the anisotropies have amplitudes $\rho_{n}$. Note that we choose the same phase factors $\theta_{n}$ as we did for the spatial anisotropy. They are related to the event planes measured experimentally. Note also the introduction of the factor 2 before $\rho_{n}$, unlike in Eq. (4).

In this paper we will restrict ourselves to anisotropies up to third order; higher orders will be omitted.

Note also that we did not include corrections to the momentum distribution due to viscosity [33, as done e.g. in [34,35. We plan to investigate this important issue in the future.

\section{Anisotropy of single-particle distributions}

The single-particle spectrum is obtained by integrating the emission function

$$
N_{1}\left(p_{t}, \phi, Y\right)=\frac{d^{3} N}{p_{t} d p_{t} d Y d \phi}=\int S(x, p) d^{4} x .
$$

The normalisation is such that the integral of $N_{1}\left(p_{t}, \phi, Y\right)$ over all momenta gives the number of particles. For the integrations of transverse directions in eq. 12 it would be convenient to use polar coordinates $r$ and $\theta$. However, it is even more convenient to use $\bar{r}$, defined in Eq. (6), instead of $r$. This requires a new Jacobian

$$
r d r d \theta=\bar{r} R^{2}(\theta) d \bar{r} d \theta .
$$

Before moving on towards the anisotropy it is interesting to explore if and how introducing spatial and flow anisotropy into the model modifies the azimuthally integrated single-particle spectrum. We have checked that if we introduce only a spatial anisotropy (i.e. the $a_{n}$ coefficients may be non-vanishing, but all $\rho_{n}$ 's are set to 0 ), then the normalisation may be slightly modified but the slope is unchanged. This is not the case for the flow anisotropy, however. As shown in Fig. 1 1 a and Fig. 1 b, flow anisotropy leads to slightly flatter spectra. We also show in Fig. 1k how the azimuthally integrated spectrum depends on the phase difference of the second and third order event planes (cf. Eq. (4))

$$
\Delta=\theta_{3}-\theta_{2} .
$$

We do not expect any correlation between the secondorder and the third-order event planes and to our knowledge there is no such correlation seen in the data. Hence, all phase differences are realised equally likely. In a data sample averaged over a large number of events the mean value of all observed curves would be measured.

Now we move on to the anisotropies of spectra which will be obtained as

$$
v_{n}=\frac{\int N_{1}\left(p_{t}, \phi, Y\right) \cos \left(n\left(\phi-\theta_{n}\right)\right) d \phi}{\int N_{1}\left(p_{t}, \phi, Y\right) d \phi} .
$$

The single-particle distributions are calculated via Eq. (12) The anisotropy coefficients $v_{n}$ can be then expressed as

$$
v_{n}\left(p_{t}\right)=\frac{C_{n}\left(p_{t}\right)}{C_{0}\left(p_{t}\right)}
$$

where

$$
\begin{aligned}
C_{n}\left(p_{t}\right) & =\int_{0}^{1} d \bar{r} \int_{0}^{2 \pi} d \theta \bar{r} R^{2}(\theta) \cos \left(n\left(\theta_{b}(\theta)-\theta_{n}\right)\right) \\
\times & I_{n}\left(\frac{p_{t} \sinh \rho(\bar{r}, \theta)}{T}\right) K_{1}\left(\frac{p_{t} \cosh \rho(\bar{r}, \theta)}{T}\right)
\end{aligned}
$$

where $I_{n}$ and $K_{1}$ are modified Bessel functions and the integration over the azimuthal angle of the momentum $\phi$ was already performed here.

The result of the calculation, however, would depend on the value of the phase difference $\Delta$. This dependence is hidden in $R(\theta)$ and $\rho(\bar{r}, \theta)$. In an experimental analysis, one effectively takes an average over all its possible values. It has been shown in 22 that the averaging may have an effect on the results. We thus have to add this averaging and introduce

$$
\begin{aligned}
\bar{C}_{n}\left(p_{t}\right) & =\int_{0}^{\frac{2}{3} \pi} d \Delta \int_{0}^{1} d \bar{r} \int_{0}^{2 \pi} d \theta \bar{r} R^{2}(\theta) \\
& \times \cos \left(n\left(\theta_{b}(\theta)-\theta_{n}\right)\right) \\
\times & I_{n}\left(\frac{p_{t} \sinh \rho(\bar{r}, \theta)}{T}\right) K_{1}\left(\frac{p_{t} \cosh \rho(\bar{r}, \theta)}{T}\right) .
\end{aligned}
$$

Then, the event-averaged $v_{n}$ is obtained as

$$
v_{n}\left(p_{t}\right)=\frac{\bar{C}_{n}\left(p_{t}\right)}{\bar{C}_{0}\left(p_{t}\right)} .
$$




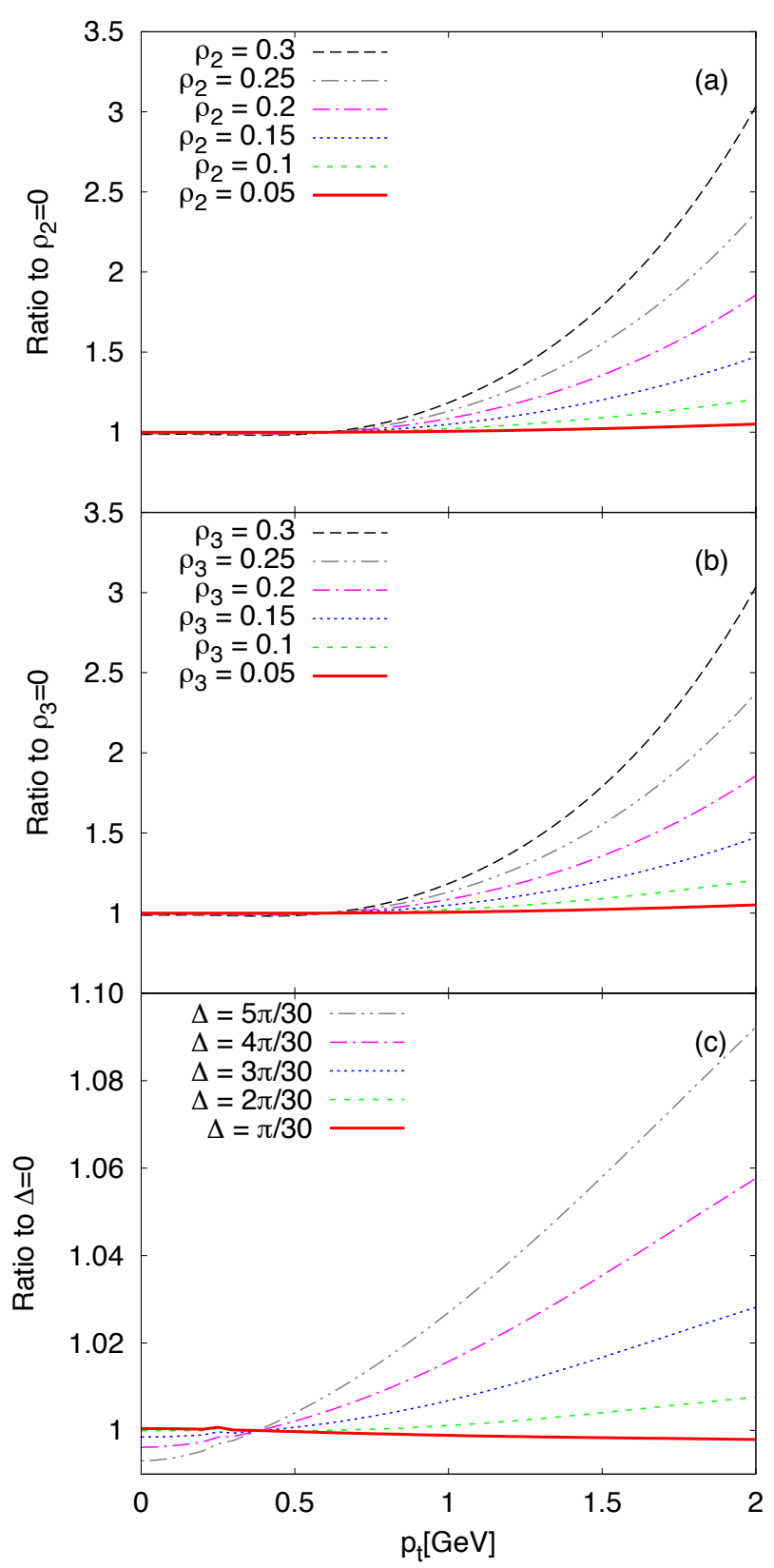

Fig. 1. The ratios of azimuthally integrated single particle spectra from fireballs with anisotropies to a reference spectrum calculated for the same set parameters except all anisotropy coefficients set to 0 . Calculated for (directly produced) pions and $T=120 \mathrm{MeV}, \rho_{0}=0.8, R_{0}=7 \mathrm{fm}, \tau_{0}=10 \mathrm{fm} / c$. (a) Ratios of spectra with second-order flow anisotropy and $a_{2}=a_{3}=\rho_{3}=0$. (b) Ratios of spectra with third-order flow anisotropy and $a_{2}=a_{3}=\rho_{2}=0$. (c) Ratios of spectra with $a_{2}=a_{3}=\rho_{2}=\rho_{3}=0.1$ and different values of $\Delta=\theta_{3}-\theta_{2}$.
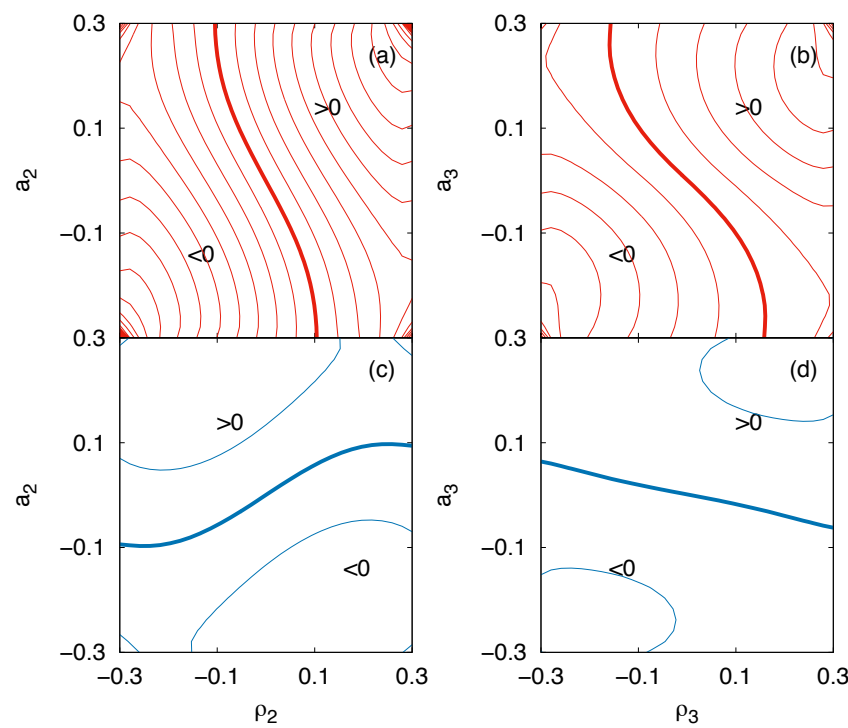

Fig. 2. Dependence of $v_{n}$ 's on spatial anisotropy $a_{n}$ and flow anisotropy $\rho_{n}$ of the same order. Results are shown for pions (upper row) and protons (lower row) at $p_{t}=300 \mathrm{MeV}$. Model parameters are $T=120 \mathrm{MeV}, \rho_{0}=0.8, R_{0}=7 \mathrm{fm}, \tau_{0}=10$ $\mathrm{fm} / c$. The thick line identify $v_{2}$ or $v_{3}$ equal to 0 , the thin lines correspond to increment or decrease of $v_{2}\left(v_{3}\right)$ by 0.005 .

We calculated the dependence of $v_{2}$ and $v_{3}$ on the anisotropy coefficients $a_{n}$ and $\rho_{n}$. We have checked that the $v_{n}$ 's of given order basically depend only on coefficients of the same order, therefore we shall only investigate such same-order dependences. In Fig. 2 we show the contour plots where dependence on both spatial and flow anisotropy can be seen. A complex structure is observed. In general, we can conclude, similarly to [19,20,22, that alone by measuring the anisotropy of single-particle distribution one is unable to determine uniquely both spatial and flow anisotropy. For heavy particles, like protons, $v_{n}$ 's seem to be driven by the spatial anisotropy. Nevertheless, flow anisotropy kicks in as the value of $a_{n}$ grows. There is even a maximum assumed by $v_{2}$ and $v_{3}$ as a function of the corresponding $a_{n}$, although it may well be beyond the phenomenologically relevant parameter region. The reason is that for high enough values of $a_{n}$ the outer surface of the fireball becomes concave and a smaller region moves transversely in the direction of the event plane. Although a unique combination of source parameters might possibly be determined from the combination of measurements with different particle species, a clear answer shall be provided by measuring the azimuthal dependence of the correlation radii.

\section{Spatial anisotropy seen by femtoscopy}

The femtoscopic technique which uses two-particle correlations is standard tool for measuring the space-time characteristics of the emitting source. Here we employ the standard formalism where the correlation function is de- 
fined as

$$
C\left(p_{1}, p_{2}\right)=\frac{N_{2}\left(p_{1}, p_{2}\right)}{N_{1}\left(p_{1}\right) N_{1}\left(p_{2}\right)}=\frac{\frac{d^{6} N}{d p_{1}^{3} d p_{2}^{3}}}{\frac{d^{3} N}{d p_{1}^{3}} \frac{d^{3} N}{d p_{2}^{3}}} .
$$

Instead of the momenta of the two particles, the correlation function is usually parametrised in terms of the average momentum $K$ and the momentum difference $q$

$$
\begin{aligned}
K & =\frac{1}{2}\left(p_{1}+p_{2}\right) \\
q & =p_{1}-p_{2} .
\end{aligned}
$$

Due to the on-shell constraint $K \cdot q=0$, the time component $q^{0}$ can be expressed as

$$
q^{0}=\frac{\boldsymbol{K}}{K^{0}} \boldsymbol{q}=\boldsymbol{\beta} \boldsymbol{q} .
$$

Hence, only three spatial components of $q$ shall be taken as independent. Note that we have introduced the pair velocity $\boldsymbol{\beta}$. The analysis is performed in the standard outside-longitudinal reference frame where the outward direction is identified with the direction of the transverse component of $\boldsymbol{K}$ and the longitudinal axis is parallel to the beam. Correlation function is then measured for $\boldsymbol{K}$ from some interval and its inverse widths in $q$ carry information about space-time structure of the source. At given $\boldsymbol{K}$-range one does not measure the size of the whole fireball but rather its homogeneity lengths. Those are the sizes of homogeneity regions. The homogeneity region is a part of the whole fireball which produces hadrons with momentum $K$ from a given range. Due to expansion it is usually smaller than the whole fireball. We shall particularly look at how the homogeneity lengths vary with the azimuthal angle $\phi$ of the $\boldsymbol{K}$ vector.

We will assume in what follows that the dependence of the correlation function on momentum difference $q$ can be reasonably well parametrised by a Gaussian prescription

$$
\begin{aligned}
C(q, K)-1= & \exp \left(-R_{o}^{2} q_{o}^{2}-R_{s}^{2} q_{s}^{2}-R_{l}^{2} q_{l}^{2}\right. \\
& \left.-2 R_{o s}^{2} q_{o} q_{s}-2 R_{o l}^{2} q_{o} q_{l}-2 R_{s l}^{2} q_{s} q_{l}\right),
\end{aligned}
$$

where $R_{o}, R_{s}, R_{l}, R_{o s}, R_{o l}$, and $R_{s l}$ are the correlation radii which can depend on $K$. They will be directly calculated from the emission function, see below.

It is important to realise that the coordinate frame in which correlations are measured is specified by the hadrons used in the measurement. It is different from any coordinate system which is attached to the fireball. The rotation between the two frames defines the explicit angular dependence of the correlation radii. In addition to this, due to collective expansion of the fireball, hadrons flying in different directions come from different parts of the fireball and carry information about their homogeneity lengths. This introduces the implicit angular dependence of the correlation radii 36 .

Generally, in the out-side-longitudinal system it can be derived that the correlation radii are given by the space- time variances as

$$
\begin{aligned}
R_{o}^{2}(K) & =\left\langle\left(\tilde{x}_{o}-\beta_{o} \tilde{t}\right)^{2}\right\rangle \\
R_{s}^{2}(K) & =\left\langle x_{s}^{2}\right\rangle \\
R_{l}^{2}(K) & =\left\langle\left(\tilde{x}_{l}-\beta_{l} \tilde{t}\right)^{2}\right\rangle \\
R_{o s}^{2}(K) & =\left\langle\left(\tilde{x}_{o}-\beta_{o} \tilde{t}\right) \tilde{x}_{s}\right\rangle \\
R_{o l}^{2}(K) & =\left\langle\left(\tilde{x}_{o}-\beta_{o} \tilde{t}\right)\left(\tilde{x}_{l}-\beta_{l} \tilde{t}\right)\right\rangle \\
R_{s l}^{2}(K) & =\left\langle\tilde{x}_{s}\left(\tilde{x}_{l}-\beta_{l} \tilde{t}\right)\right\rangle .
\end{aligned}
$$

Note that the space-time variances depend on average momentum $K$. Here we have introduced the averaging over the source

$$
\langle f(x)\rangle=\frac{\int S(x, p) f(x) d^{4} x}{\int S(x, p) d^{4} x}
$$

and we also introduced the shifted coordinates as

$$
\tilde{x}^{\mu}=x^{\mu}-\left\langle x^{\mu}\right\rangle .
$$

Recall that the coordinates $x_{o}, x_{s}, x_{l}$ are connected with the direction of the emitted particles. The explicit angular dependence is obtained simply by expressing the out-side-longitudinal coordinates in terms of the coordinates $x, y$, which are fixed with the fireball:

$$
\begin{aligned}
& \tilde{x}_{o}=\tilde{x} \cos \phi+\tilde{y} \sin \phi \\
& \tilde{x}_{s}=-\tilde{x} \sin \phi+\tilde{y} \cos \phi .
\end{aligned}
$$

where $\phi$ is the azimuthal angle of the emitted hadron pairs. This leads to

$$
\begin{aligned}
R_{s}^{2}= & \frac{1}{2}\left(\left\langle\tilde{x}^{2}\right\rangle+\left\langle\tilde{y}^{2}\right\rangle\right)+\frac{1}{2}\left(\left\langle\tilde{x}^{2}\right\rangle-\left\langle\tilde{y}^{2}\right\rangle\right) \cos 2 \phi \\
& -\langle\tilde{x} \tilde{y}\rangle \sin 2 \phi \\
R_{o}^{2}= & \frac{1}{2}\left(\left\langle\tilde{x}^{2}\right\rangle+\left\langle\tilde{y}^{2}\right\rangle\right)-\frac{1}{2}\left(\left\langle\tilde{y}^{2}\right\rangle-\left\langle\tilde{x}^{2}\right\rangle\right) \cos 2 \phi \\
& +\langle\tilde{x} \tilde{y}\rangle \sin 2 \phi+\beta_{o}^{2}\left\langle\tilde{t}^{2}\right\rangle-2 \beta_{o}\langle\tilde{x} \tilde{t}\rangle \cos \phi \\
& -2 \beta_{o}\langle\tilde{y} \tilde{t}\rangle \sin \phi \\
R_{l}^{2}= & \left\langle\left(\tilde{z}-\beta_{l} \tilde{t}\right)^{2}\right\rangle \\
R_{o s}^{2}= & \langle\tilde{x} \tilde{y}\rangle \cos 2 \phi+\frac{1}{2}\left(\left\langle\tilde{y}^{2}\right\rangle-\left\langle\tilde{x}^{2}\right\rangle\right) \sin 2 \phi \\
& +\beta_{o}\langle\tilde{x} \tilde{t}\rangle \sin \phi-\beta_{o}\langle\tilde{y} \tilde{t}\rangle \cos \phi \\
R_{o l}^{2}= & \left\langle\left(\tilde{z}-\beta_{l} \tilde{t}\right) \tilde{x}\right\rangle \cos \phi+\left\langle\left(\tilde{z}-\beta_{l} \tilde{t}\right) \tilde{y}\right\rangle \sin \phi \\
& -\beta_{l}\left\langle\left(\tilde{z}-\beta_{l} \tilde{t}\right) \tilde{t}\right\rangle \\
R_{s l}^{2}= & \left\langle\left(\tilde{z}-\beta_{l} \tilde{t}\right) \tilde{y}\right\rangle \cos \phi-\left\langle\left(\tilde{z}-\beta_{l} \tilde{t}\right) \tilde{x}\right\rangle \sin \phi .
\end{aligned}
$$

In what follows we want to study the azimuthal dependence of the correlation radii. To this end, they are customarily expanded into Fourier series. The space-time covariances also depend non-trivially on $\phi$, although we have suppressed writing this out explicitly. They can be written out and inserted in the right-hand sides of Eqs. (28). Then, by combining all sine and cosine terms on the r.h.s. of the obtained equations one can analytically identify all terms of Fourier expansion of the correlation radii. This 
has been done earlier for the second order 36,37 . The results of the third and higher orders are presented in Appendix C.

In practical calculation, however, one can proceed differently. The whole azimuthal dependence can be calculated from Eqs. 28a $-(28 f)$. Then one can extract any Fourier coefficient from the result. With increasing complexity of higher order terms this procedure appears computationally more efficient.

Moreover, these are not yet the correlation radii which correspond to the measured ones, even if the assumption of Gaussian correlation function is valid. In order to measure the correlation radii in real collisions, hadron pairs must be collected over a large number of events. The events must be rotated so that the event planes are all aligned. Otherwise the azimuthal dependence would be averaged out. For measuring the second-order oscillations one rotates the events so as to align the second-order event planes. For the third-order oscillation one aligns the thirdorder event planes. Since the event planes of different orders may be assumed to be uncorrelated these alignments effectively introduce averaging over the direction of the other event planes. This must be included in calculations. It has been investigated in 22 that this averaging may introduce a few percent effect on the resulting correlation radii. Hence, when calculating the correlation radii from Eqs. (28), one additional integral over $\theta_{2}$ or $\theta_{3}$ must be calculated, depending on which order of oscillations we shall be interested in. We include this averaging over other event planes in our calculation.

Therefore, the second order and the third order Fourier amplitudes that we are going to calculate do not belong to the same Fourier series. In the former case the emission function is averaged over all possible values of $\theta_{3}$, in the latter averaging runs over $\theta_{2}$. This will be also reflected in notation: the correlation radii will be expanded into series

$$
R_{i}^{2}(\phi)=R_{i, j, 0}^{2}+\sum_{n=1}^{\infty} R_{i, j, n}^{2} \cos \left(n\left(\phi-\phi_{n}\right)\right)
$$

where $i=o, s$, and $j=2,3$, depending on which event plane $\theta_{j}$ has been put to 0 . In general, terms of the same order may differ, if they come from averaging with different event-planes fixed. For example, we note that

$$
\begin{aligned}
& R_{o, 2,0}^{2} \neq R_{o, 3,0}^{2} \\
& R_{s, 2,0}^{2} \neq R_{s, 3,0}^{2} .
\end{aligned}
$$

Second order oscillations have been calculated in 19 , but the averaging over third order event plane was not performed there. In order to fill this gap, we have done the calculation here and show the results in Fig. 3 The most important parameter which sets the scale of the transverse correlation radii is $R_{0}$. We can see that even the average radii $R_{i, 2,0}^{2}$ depend on both anisotropy parameters $a_{2}$ and $\rho_{2}$. For the higher-order Fourier terms, we would like to factorise out their trivial scaling with $R_{0}$, thus for the analysis we divide all amplitudes by the zeroth-order term. As it was observed previously 19, the

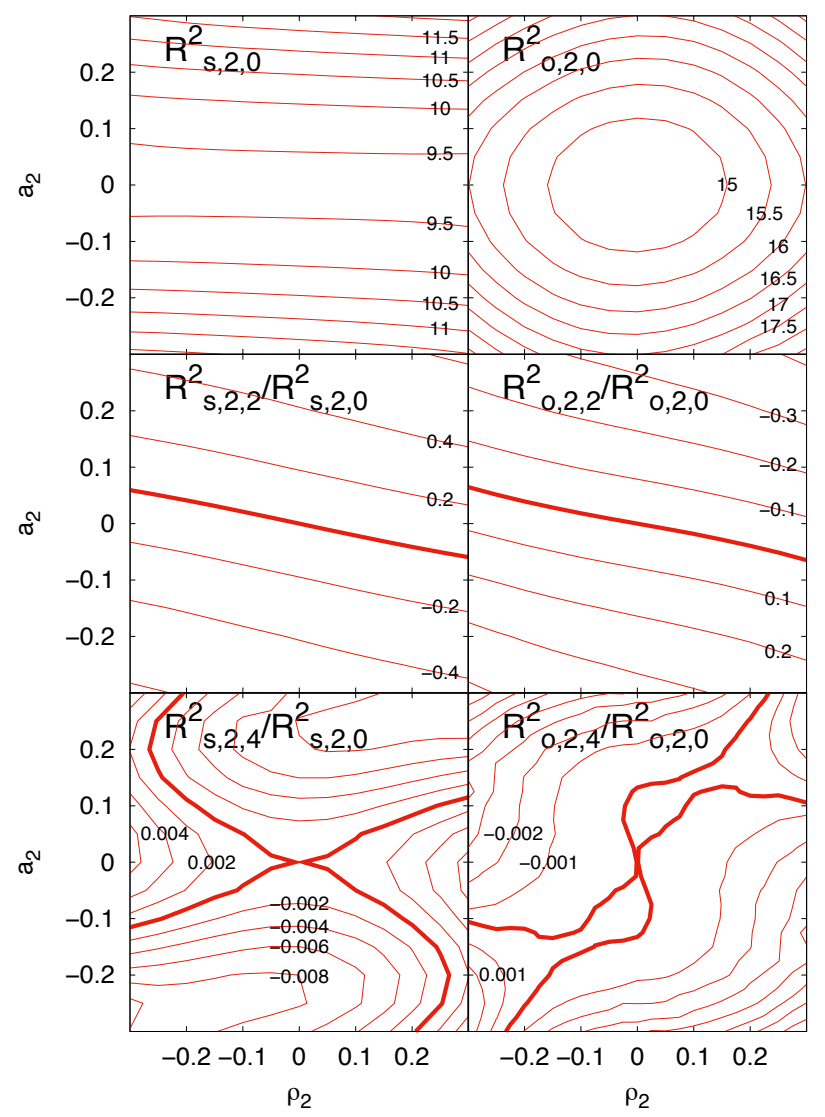

Fig. 3. The dependence of transverse correlation radii and their oscillation amplitudes on second-order anisotropies in space and transverse flow. The values are calculated for outward pair momentum $K_{o}=300 \mathrm{MeV}$. Model parameters used in the calculation are $T=120 \mathrm{MeV}, \rho_{0}=0.8, R_{0}=7 \mathrm{fm}$, $\tau_{0}=10 \mathrm{fm} / c$. Third-order anisotropy parameters were set to $a_{3}=\rho_{3}=0.1$.

second order oscillation amplitude is mainly set by the spatial anisotropy parameter $a_{2}$. The dependence on $\rho_{2}$ is weak. This confirms the early conjecture that the secondorder spatial deformation can be measured with the help of correlation radii [19. Both spatial and flow anisotropy can then be obtained from combined measurement of $v_{2}$ and the azimuthal dependence of correlation radii. Figure 3 also shows that in this model higher-order terms in the decomposition of the correlation radii are very small. The fourth-order terms are smaller than the second-order terms by two orders of magnitude. Although it shows an interesting dependence on $a_{2}$ and $\rho_{2}$, it is most likely below any reasonable experimental sensitivity.

We have also looked at third-order oscillation in case of averaging over all possible directions of the second-order event plane. The resulting dependence of the correlation radii on $a_{3}$ and $\rho_{3}$ is plotted in Fig. 4

Again, even the azimuthally averaged radii show some dependence on both anisotropy parameters. The third- 


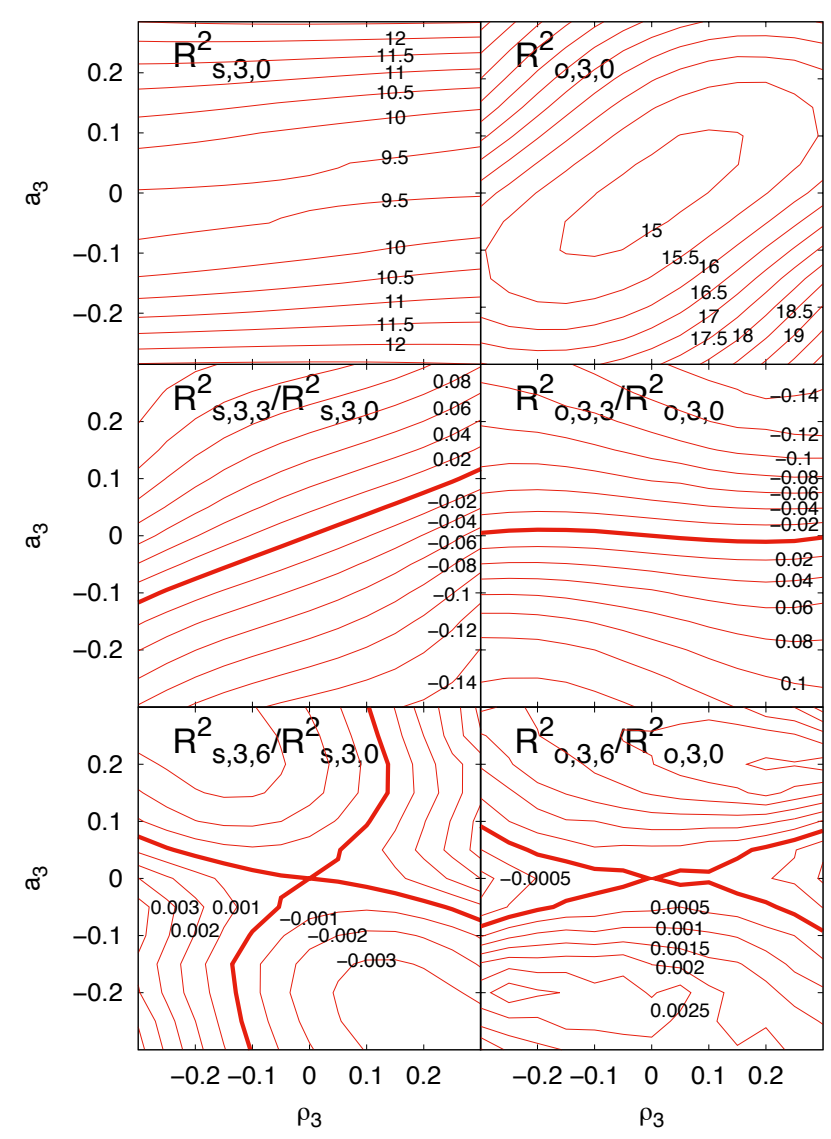

Fig. 4. The dependence of transverse correlation radii and their oscillation amplitudes on third-order anisotropies in space and transverse flow. The values are calculated for outward pair momentum $K_{o}=300 \mathrm{MeV}$. Model parameters used in the calculation are $T=120 \mathrm{MeV}, \rho_{0}=0.8, R_{0}=7 \mathrm{fm}, \tau_{0}=10$ $\mathrm{fm} / c$. Second-order anisotropy parameters were set to $a_{2}=$ $\rho_{2}=0.1$.

order spatial anisotropy is best reflected in the third-order scaled amplitude of the outward radii $R_{o, 3,3}^{2} / R_{o, 3,0}^{2}$. For the sideward radius the third-order oscillation depends on both $a_{3}$ and $\rho_{3}$. Note, however, that the third-order oscillation is typically smaller by an order of magnitude if it is compared to the second-order oscillation in Fig. 3 Even more suppressed is the next higher order, which is the sixth in this case. In absolute numbers the scaled amplitudes are on the level of a few per mille or even less. We do not expect that such a weak signal could be reasonably measured in experiments.

\section{Relation to data}

The present model has been designed with the aim to better characterise measured data on spectra and anisotropies. However, such an analysis requires to take into account many more issues and is technically much more involved.

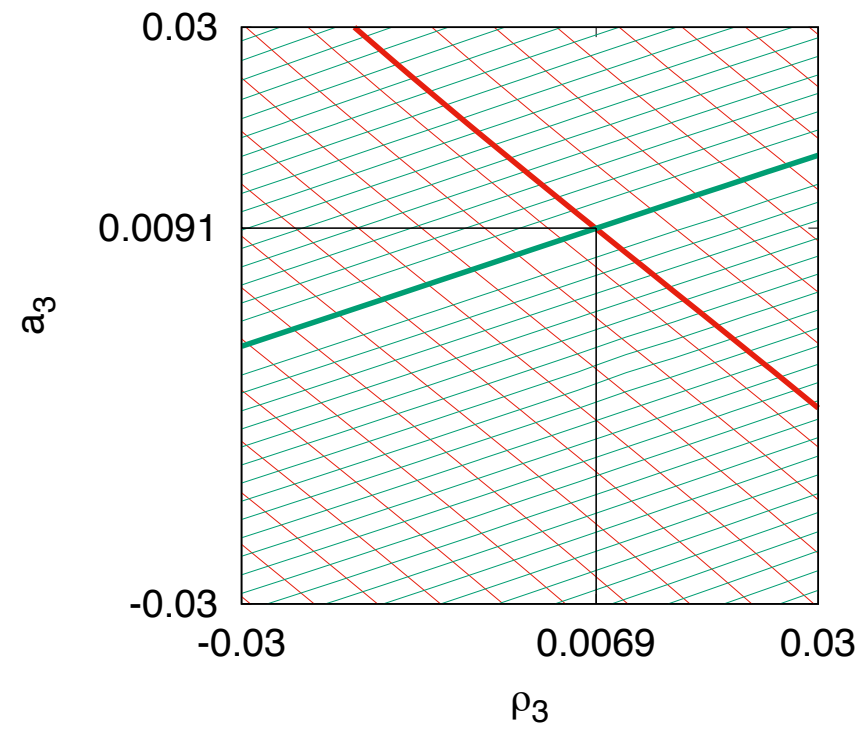

Fig. 5. Combined contour lines of constant $v_{3}$ (red from top left to bottom right) and constant $R_{s, 3,3}^{2} / R_{s, 0,3}^{2}$ (green from bottom left to top right). Thick lines show the values of data for $p_{t}=863 \mathrm{MeV}\left(v_{3}\right)$ [0] and $K_{t}=877 \mathrm{MeV}$ (correlation radii) 26]. The increment between neighbouring lines is 0.01 .

In order to reach physically relevant results, resonances must be included in the analysis [38. This highly increases the complexity of calculations. Then, all data, i.e. identified spectra, anisotropy coefficients, and correlations, should be fitted simultaneously. The new Bayesian technique [18 seems well suitable to this aim. Such a thorough analysis, however, goes far beyond our scope here. Nevertheless, we want to illustrate the qualitative features presented in previous sections with the help of comparison to data. We hasten to stress that this comparison should be understood merely on qualitative level.

The STAR Collaboration has measured data from $\mathrm{Au}+\mathrm{Au}$ collisions at $\sqrt{s_{N N}}=200 \mathrm{GeV}$. They have analysed secondorder oscillations of correlation radii as functions of azimuthal angle with the blast-wave model extended to that order in 39. In that analysis, the temperature $T$ and transverse flow gradient $\rho_{0}$ were inferred from a simultaneous fit to pion, kaon, and proton $p_{t}$ spectra and $v_{2}$. Analysis of the azimuthally sensitive correlation radii yielded the sizes of the fireball and the second-order anisotropy parameters. From this analysis of 10-20\% centrality STAR data, the following model parameter values have been extracted by the STAR Collaboration 39: $T=98 \mathrm{MeV}$, $\rho_{0}=0.98, \rho_{2}=0.05, \tau_{0}=7.8 \mathrm{fm} / c, \Delta \tau=2.59 \mathrm{fm} / c$. The second-order spatial anisotropy can be translated into our model as $R_{0}=11.4 \mathrm{fm}, a_{2}=0.0439$.

With these values fixed we calculate the combined contour plot for the dependences of $v_{3}$ and third-order scaled amplitude $R_{s, 3,3}^{2} / R_{s, 3,0}^{2}$ on $a_{3}$ and $\rho_{3}$. It is shown in Fig. 5 The curves decreasing to the right are lines with constant $v_{3}$, the others correspond to constant $R_{s, 3,3}^{2} / R_{s, 0,3}^{2}$. Thick lines represent the data values by PHENIX [40,26]. Out of available data we had to choose bins in $p_{t}$ for $v_{3}$ and 


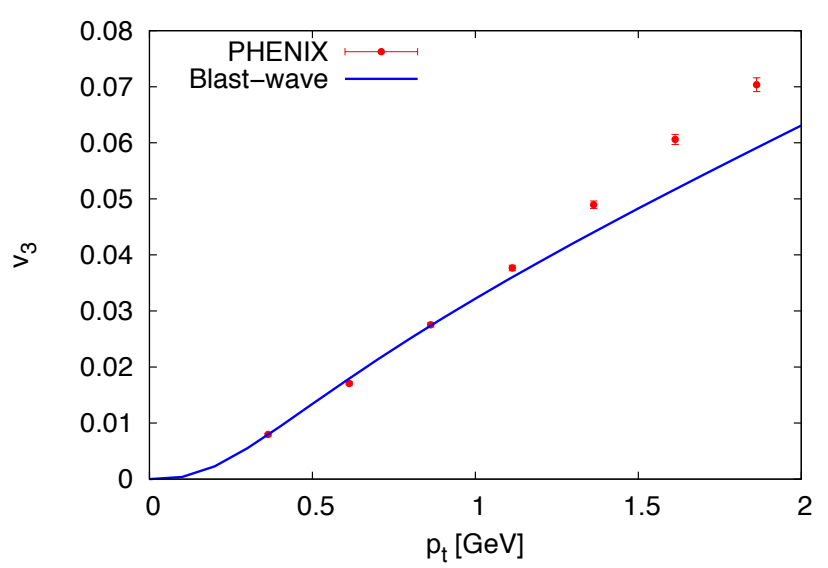

Fig. 6. The $p_{t}$ dependence of $v_{3}$ for identified pions measured by PHENIX collaboration [40] compared with the theoretical curve from the blast-wave model with parameters determined in Fig. 5.

correlation radii, which overlap. Unfortunately, these two sets of data are measured in slightly different momentum ranges. In order to use overlapping $p_{t}$ bins, we have taken $v_{3}$ point for pions at $p_{t}=863 \mathrm{MeV}$ [40 and correlation radii for $K_{t}=877 \mathrm{MeV}[26$. The values extracted from such a simple comparison with data are: $a_{3}=0.0091$ and $\rho_{3}=0.0069$.

With the extracted model parameters we tried to calculate theoretical predictions for $v_{3}$ (Fig. 6) and the azimuthal angle dependence of the correlation radii (Fig. 7).

For the latter we chose to plot the radii measured for $K_{t}=530 \mathrm{MeV} / c$ as was also done by the PHENIX Collaboration in [26]. The model fails completely in reproducing the absolute size of the correlation radii. The corresponding parameter, however, has been fixed from the STAR analysis of second-order oscillations of correlation radii. The mean values of the radii should be very similar for second and the third order oscillations. This clearly demonstrates the need of simultaneous fit to all available data in single analysis if one wants to go beyond the qualitative level.

We also show in Fig. 8 the third-order scaled amplitudes of correlation radii as functions of $K_{t}$. The experimental error bars are huge and we explained that we have based our analysis on the point at highest $K_{t}$. It seems that the PHENIX data require amplitudes of opposite signs, which the blast-wave model can accommodate.

We want to close this section with a few comments on the applicability of the model to preliminary ALICE data from $\mathrm{Pb}+\mathrm{Pb}$ collisions at $\sqrt{s_{N N}}=2.76 \mathrm{TeV}$ 41. The data seem to indicate that the third-order oscillation amplitudes of both $R_{s}^{2}$ and $R_{o}^{2}$ are negative. By inspecting Fig. 4 we find that such situation only happens in a small region in parameter space with $a_{3}$ around 0 and positive $\rho_{3}$. Thus from combined measurements of outward and sideward radii one could deduce that the fireball at the

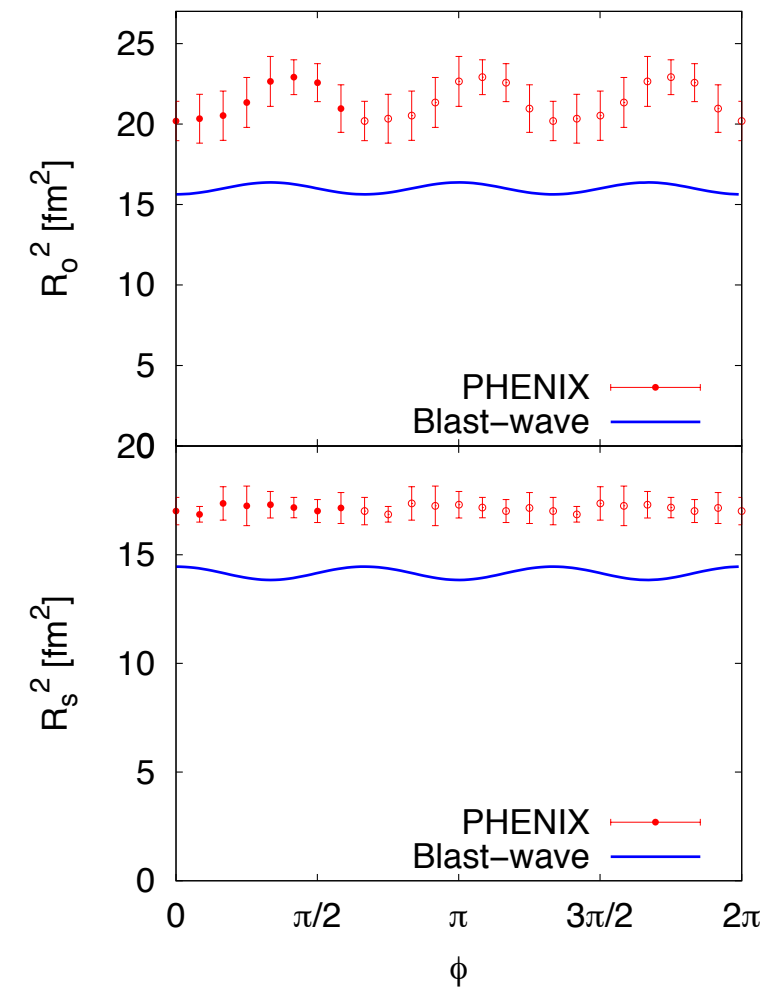

Fig. 7. The azimuthal dependence of correlation radii with respect to the third-order event plane for $K_{t}=530 \mathrm{MeV} / c$. Data by PHENIX collaboration 26 are compared with results from the blast-wave model with parameters determined in Fig. 5.

LHC has rather symmetric shape and the anisotropy is set by the transverse collective velocity field.

\section{Conclusions}

We have generalised the blast-wave model so that it includes third-order anisotropies in both space and expansion. Analogically to the second order, from the combination of Fig. 2 and Fig. 4 we can infer, that it is indeed possible to reconstruct both anisotropy coefficients of this model: $a_{3}$ and $\rho_{3}$, from measurements of the azimuthal anisotropy of single-particle momentum distributions and HBT radii.

We have also pointed out the need for averaging over the difference of second and third-order reaction planes when focusing on a selected order of Fourier decomposition of the hadron distribution or the correlation radii. This is effectively done in data analysis when all events are aligned according to the event plane of the selected order.

The contour plots shown in Fig. 4 exemplify the statement made in 21 that at fixed flow anisotropy the amplitude of correlation radii oscillation can be tuned with the help of spatial anisotropy and even a flip in the phase can be obtained. Such a flip of the phase corresponds to a change of the sign of the amplitude. Keeping constant flow 


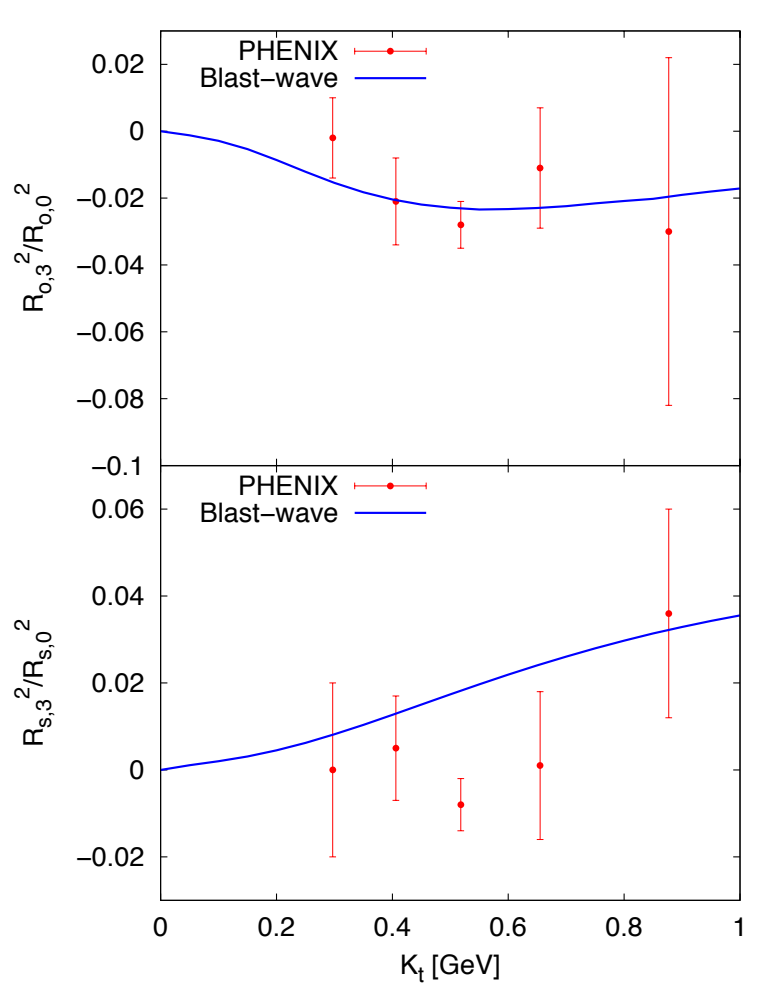

Fig. 8. The $K_{t}$ dependence of third-order oscillation amplitude of the correlation radii. Data by PHENIX collaboration 26] are compared with results from the blast-wave model with parameters determined in Fig. 5.

anisotropy and changing space anisotropy corresponds to moving vertically in the panels of Fig. 4 and the phase flip corresponds to crossing the thick line in that Figure. Our results are much more detailed since we show the full dependence on the two parameters.

We have also calculated the subleading terms for the third-order anisotropies in the oscillations of correlation radii. These are the sixth-order oscillations. They were shown to be an order of magnitude smaller than the lower order and hardly measurable at current experimental statistics. We have actually derived expressions for oscillation amplitudes at general order, but there is currently no need to go to higher orders also with model studies as sufficient statistics would hardly be available.

It is interesting to compare our results to those obtained in an analogical study which used the Buda-Lund model 222. In that model, the way in which observables depend on the combination of the space and flow anisotropy is different to the one presented here. Partially, this is due to our definition of the spatial profile with the minus sign in Eq. (4). The corresponding sign in the BudaLund model was kept to be plus. On the qualitative level, the oscillations of the correlation radii within the BudaLund model seem to be much more sensitive to the flow anisotropy than it is the case in this study.

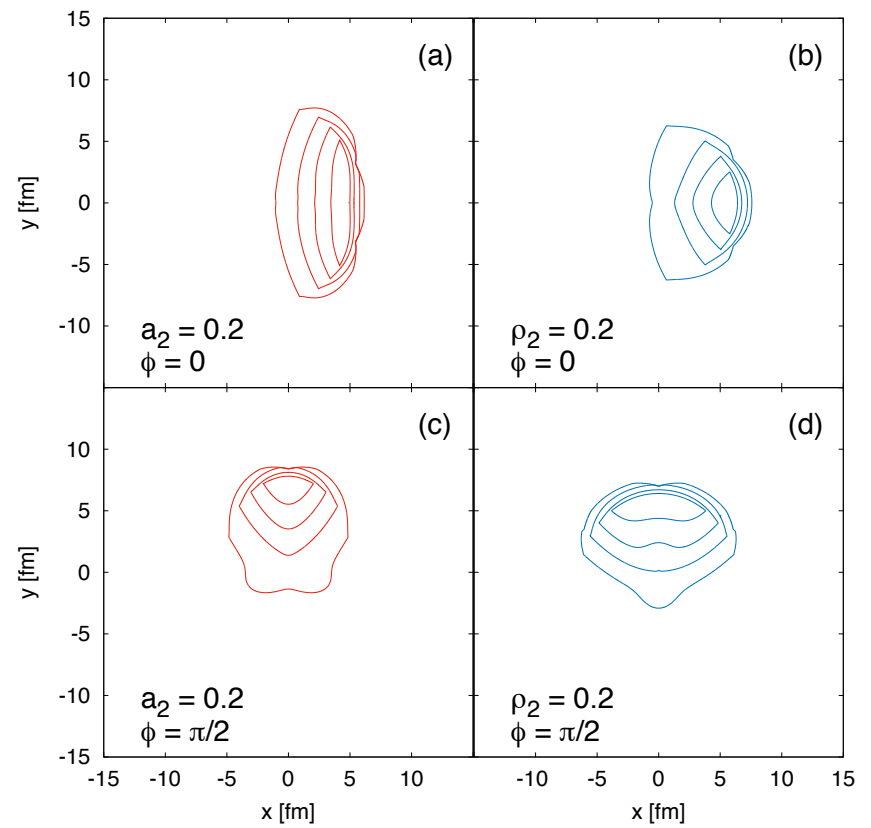

Fig. 9. Transverse profiles of the emission function for the blast-wave model with source parameters as in Fig. 4. for particles with $p_{t}=300 \mathrm{MeV}$ in the indicated direction $\phi$. Left column: only density profile anisotropy; right column: only flow anisotropy. Other source parameters are $T=120 \mathrm{MeV}$, $\tau_{0}=10 \mathrm{fm}, R_{0}=7 \mathrm{fm}, \Delta \tau=1 \mathrm{fm}, \rho_{0}=0.8$. Shown are the effective sources which are calculated by integrating over all directions of the third-order anisotropy with $a_{3}=\rho_{3}=0.1$. The contours correspond to levels of $0.8,0.6,0.4$, and 0.2 of the maximum.

In order to explore the difference of the two models in more details, in Figs. 9 and 10 we plot transverse profiles of the emitting sources according to the blast-wave and Buda-Lund models, respectively. Plotted are not the emission functions directly. We have assumed that there are the third-order anisotropies, as well, which have their third-order event planes completely independent from the second-order event plane directions. Then, we have integrated over all possible directions of the third-order event plane and obtained an effective emission function with only second-order anisotropy. In the figures, we have assumed pions with $p_{t}=300 \mathrm{MeV}$ and two different azimuthal angles of particle emission: $\phi=0$ (upper rows) and $\phi=\pi / 2$ (lower rows).

Figures 9 and 10 clearly demonstrate that the two models behave rather differently. In the Buda-Lund model, the deformations caused by the density anisotropy (Fig. 10 left) and by flow anisotropy (Fig. 10 right) look qualitatively similar. On the other hand, the sharp source boundary in the blast wave model has severe influence. The spatial anisotropy (Fig. 9 left) makes the two sources for $\phi=0$ and $\phi=\pi / 2$ qualitatively much more different from each other than the flow anisotropy (Fig. 9 right). Note that the sources should be compared in the out-side-long coordinate system, and the outward axis is directed in the 


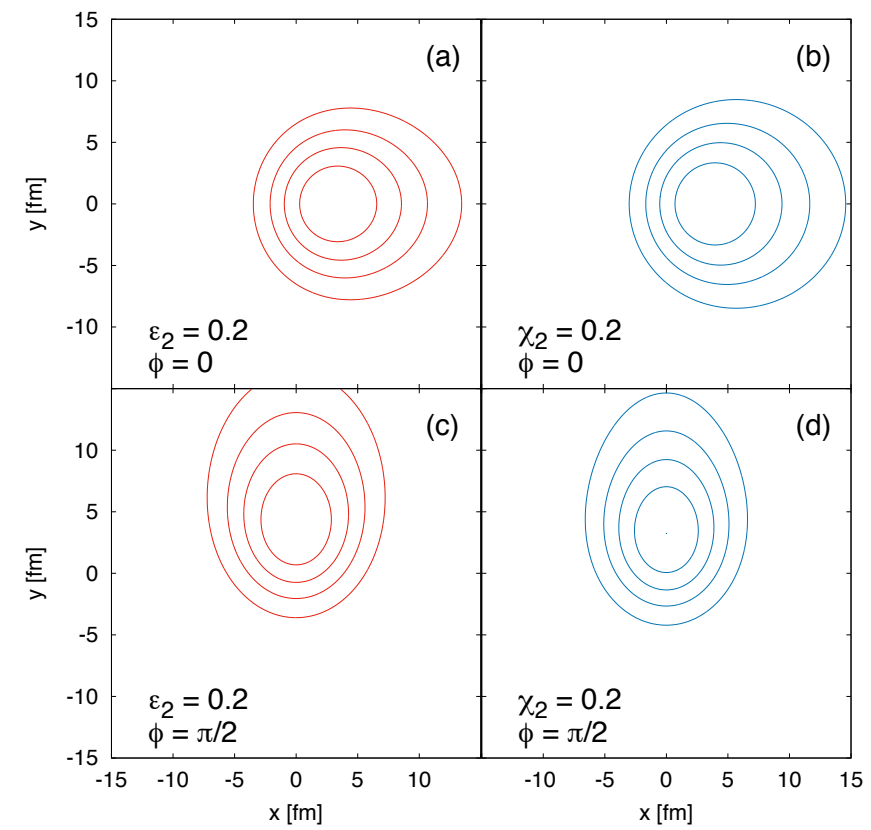

Fig. 10. Analogical to Fig. 9, but calculated with the BudaLund model, with source parameters $T=120 \mathrm{MeV}, \tau_{0}=$ $10 \mathrm{fm}, a_{2}=0.3, b=-0.1, R=7, Z=15, H=8, H_{z}=16$, $\epsilon_{3}=\chi_{3}=0.1$. The model is explained in 22 . The values of the second-order anisotropy parameters are indicated in the figure. The averaging over all directions of the third-order anisotropy is performed with $\varepsilon_{3}=\chi_{3}=0.1$.

direction of $\phi$. Thus we see the much stronger dependence on shape anisotropy than on flow anisotropy.

Having the two models which appear so differently, the choice of the more suitable model should be decided by data. Note, however, that neither this schematic study, nor the one of ref. 22] included meson production from the decays of resonances and the modification of the correlation function due to viscosity. Their influence should be investigated in the future in order to arrive at a conclusive answer.

This work has been performed in framework of COST Action CA15213 "Theory of hot matter and relativistic heavy-ion collisions" (THOR). Partial support by VEGA 1/0469/15 (Slovakia) and by MŠMT grant No. LG15001 (Czech Republic) is acknowledged. M. Cs. was supported by the János Bolyai Research Scholarship of the Hungarian Academy of Sciences.

\section{A Relation between different parametrisations of second-order spatial anisotropy}

In this Appendix we derive the relation between our parametrisation of the transverse shape and the one used in [19].

First of all, it should be clearly stated that the two parametrisations are different. Hence, the elliptic shape that has been used in 19 would be fully reproduced with the help of parametrisation (4) only if higher-order terms are included. Of course, the importance of higher orders drops with $n$.

We shall assume here that the second-order anisotropy is small, i.e., the parameter $a$ from Eq. (5) is close to 1 . The ellipse of [19] includes points with coordinates

$$
\begin{aligned}
& r_{x}=R_{x} \cos \theta=a R^{\prime} \cos \theta \\
& r_{y}=R_{y} \sin \theta=\frac{R^{\prime}}{a} \sin \theta .
\end{aligned}
$$

Thus the radius as function of the azimuthal angle is

$$
R=\sqrt{r_{x}^{2}+r_{y}^{2}}=\sqrt{a^{2} R^{\prime 2} \cos ^{2} \theta+\frac{R^{\prime 2}}{a^{2}} \sin ^{2} \theta} .
$$

This can be rewritten as

$$
R=R^{\prime} \sqrt{\frac{a^{4}+1}{2 a^{2}}}\left(1+\frac{a^{4}-1}{a^{4}+1} \cos (2 \theta)\right)^{\frac{1}{2}} .
$$

Now we assume that $a \rightarrow 1$ and therefore $\left(a^{4}-1\right) /\left(a^{4}+1\right)$ is very small. Thus we can Taylor-expand the bracket up to first order and obtain

$$
R(\theta) \approx R^{\prime} \sqrt{\frac{a^{4}+1}{2 a^{2}}}\left(1+\frac{1}{2} \frac{a^{4}-1}{a^{4}+1} \cos (2 \theta)\right) .
$$

The mean radius $R_{0}$ of the present model is to be identified with

$$
R_{0}=R^{\prime} \sqrt{\frac{a^{4}+1}{2 a^{2}}} .
$$

The amplitude of the oscillations is to be identified with $\left(-a_{2}\right)$

$$
a_{2}=-\frac{1}{2} \frac{a^{4}-1}{a^{4}+1}
$$

Inverting this relation gives

$$
a=\left(\frac{1-2 a_{2}}{1+2 a_{2}}\right)^{\frac{1}{4}} .
$$

\section{B The direction of transverse velocity}

Here we derive the direction of the transverse velocity, which is given by the angle $\theta_{b}$. Since it is supposed to be perpendicular to the surface of constant $\bar{r}$, we write down its coordinates

$$
\begin{aligned}
& x=\bar{r} R_{0}\left(1-\sum_{n=2}^{\infty} a_{n} \cos \left(n\left(\theta-\theta_{n}\right)\right)\right) \cos \theta \\
& y=\bar{r} R_{0}\left(1-\sum_{n=2}^{\infty} a_{n} \cos \left(n\left(\theta-\theta_{n}\right)\right)\right) \sin \theta .
\end{aligned}
$$

In further calculations, however, we shall truncate the expansion after the third-order term. 
The (truncated) expressions $(38)$ can be inserted into the derivatives in Eq. 10. This gives

$$
\theta_{b}=\frac{\pi}{2}+\arctan \frac{A}{B}
$$

where

$$
\begin{array}{r}
A=4 a_{2} \sin \theta+3 a_{3} \frac{\sin 3\left(\theta-\theta_{3}\right)}{\cos \theta} \\
-\frac{-1+a_{2} \cos 2\left(\theta-\theta_{2}\right)+a_{3} \cos 3\left(\theta-\theta_{3}\right)}{\sin \theta} \\
B=a_{2} \cos \theta+3 a_{3} \frac{\sin 3\left(\theta-\theta_{3}\right)}{\sin \theta} \\
\quad-\frac{-1+a_{2} \cos 2\left(\theta-\theta_{2}\right)+a_{3} \cos 3\left(\theta-\theta_{3}\right)}{\cos \theta}
\end{array}
$$

\section{Fourier amplitudes of the correlation radii}

Here we give an overview of the Fourier amplitudes of the azimuthal dependence of outward, sideward, and outside cross-term correlation radii. Note that these formulas are model-independent. Dependence on a particular model comes into evaluation of individual space-time (co)variances. Note that analogical relations have been derived in [21, where Milne coordinates were used instead of the Cartesian ones.

We define the amplitudes

$$
\begin{aligned}
& R_{o}^{2}=\left(R_{o}^{2}\right)_{0}+\sum_{n=1}^{\infty}\left[\left(R_{o}^{2}\right)_{n}^{s} \sin (n \phi)+\left(R_{o}^{2}\right)_{n}^{c} \cos (n \phi)\right] \\
& R_{s}^{2}=\left(R_{s}^{2}\right)_{0}+\sum_{n=1}^{\infty}\left[\left(R_{s}^{2}\right)_{n}^{s} \sin (n \phi)+\left(R_{s}^{2}\right)_{n}^{c} \cos (n \phi)\right] \\
& R_{o s}^{2}=\left(R_{o s}^{2}\right)_{0}+\sum_{n=1}^{\infty}\left[\left(R_{o s}^{2}\right)_{n}^{s} \sin (n \phi)+\left(R_{o s}^{2}\right)_{n}^{c} \cos (n \phi)\right]
\end{aligned}
$$

In a similar way we shall expand the space-time (co-)variances

$$
\begin{aligned}
\left\langle\tilde{x}^{\mu} \tilde{x}^{\nu}\right\rangle & =\left\langle\tilde{x}^{\mu} \tilde{x}^{\nu}\right\rangle_{0} \\
& +\sum_{n=1}^{\infty}\left[\left\langle\tilde{x}^{\mu} \tilde{x}^{\nu}\right\rangle_{n}^{s} \sin (n \phi)+\left\langle\tilde{x}^{\mu} \tilde{x}^{\nu}\right\rangle_{n}^{c} \cos (n \phi)\right]
\end{aligned}
$$

The series (43) are inserted into the model-independent expressions (28) and the sine and cosine terms are reorganised with the help of addition theorems. Then one can collect the terms order by order and put them in equality with the corresponding expansion of the correlation radii.
We obtain for the outward radius

$$
\begin{aligned}
& \left(R_{o}^{2}\right)_{0}=\frac{1}{2}\left\langle\tilde{x}^{2}\right\rangle_{0}+\frac{1}{2}\left\langle\tilde{y}^{2}\right\rangle_{0}-\frac{1}{4}\left\langle\tilde{y}^{2}\right\rangle_{2}^{c}+\frac{1}{4}\left\langle\tilde{x}^{2}\right\rangle_{2}^{c} \\
& +\frac{1}{2}\langle\tilde{x} \tilde{y}\rangle_{2}^{s}+\beta_{o}^{2}\left\langle\tilde{t}^{2}\right\rangle_{0}-\beta_{o}\langle\tilde{t} \tilde{x}\rangle_{1}^{c} \\
& -\beta_{o}\langle\tilde{t} \tilde{y}\rangle_{1}^{s} \\
& \left(R_{o}^{2}\right)_{1}^{c}=\frac{3}{4}\left\langle\tilde{x}^{2}\right\rangle_{1}^{c}+\frac{1}{4}\left\langle\tilde{y}^{2}\right\rangle_{1}^{c}-\frac{1}{4}\left\langle\tilde{y}^{2}\right\rangle_{3}^{c}+\frac{1}{4}\left\langle\tilde{x}^{2}\right\rangle_{3}^{c} \\
& +\frac{1}{2}\left(\langle\tilde{x} \tilde{y}\rangle_{1}^{s}+\langle\tilde{x} \tilde{y}\rangle_{3}^{s}\right)+\beta_{o}^{2}\left\langle\tilde{t}^{2}\right\rangle_{1}^{c}-2 \beta_{o}\langle\tilde{t} \tilde{x}\rangle_{0} \\
& -\beta_{o}\langle\tilde{t} \tilde{x}\rangle_{2}^{c}-\beta_{o}\langle\tilde{t} \tilde{y}\rangle_{2}^{s} \\
& \left(R_{o}^{2}\right)_{1}^{s}=\frac{1}{4}\left\langle\tilde{x}^{2}\right\rangle_{1}^{s}+\frac{3}{4}\left\langle\tilde{y}^{2}\right\rangle_{1}^{s}+\frac{1}{4}\left\langle\tilde{y}^{2}\right\rangle_{3}^{s}-\frac{1}{4}\left\langle\tilde{x}^{2}\right\rangle_{3}^{s} \\
& +\frac{1}{2}\left(\langle\tilde{x} \tilde{y}\rangle_{1}^{c}-\langle\tilde{x} \tilde{y}\rangle_{3}^{c}\right)+\beta_{o}^{2}\left\langle\tilde{t}^{2}\right\rangle_{1}^{s}-\beta_{o}\langle\tilde{t} \tilde{x}\rangle_{2}^{s} \\
& -2 \beta_{o}\langle\tilde{t} \tilde{y}\rangle_{0}+\beta_{o}\langle\tilde{t} \tilde{y}\rangle_{2}^{c} \\
& \left(R_{o}^{2}\right)_{2}^{c}=\frac{1}{2}\left\langle\tilde{x}^{2}\right\rangle_{2}^{c}+\frac{1}{2}\left\langle\tilde{y}^{2}\right\rangle_{2}^{c}-\frac{1}{2}\left\langle\tilde{y}^{2}\right\rangle_{0}-\frac{1}{4}\left\langle\tilde{y}^{2}\right\rangle_{4}^{c} \\
& +\frac{1}{2}\left\langle\tilde{x}^{2}\right\rangle_{0}+\frac{1}{4}\left\langle\tilde{x}^{2}\right\rangle_{4}^{c}+\frac{1}{2}\langle\tilde{x} \tilde{y}\rangle_{4}^{s}+\beta_{o}^{2}\left\langle\tilde{t}^{2}\right\rangle_{2}^{c} \\
& -\beta_{o}\langle\tilde{t} \tilde{x}\rangle_{3}^{c}-\beta_{o}\langle\tilde{t} \tilde{x}\rangle_{1}^{c}-\beta_{o}\langle\tilde{t} \tilde{y}\rangle_{3}^{s} \\
& +\beta_{o}\langle\tilde{t} \tilde{y}\rangle_{1}^{s} \\
& \left(R_{o}^{2}\right)_{2}^{s}=\frac{1}{2}\left\langle\tilde{x}^{2}\right\rangle_{2}^{s}+\frac{1}{2}\left\langle\tilde{y}^{2}\right\rangle_{2}^{s}+\frac{1}{4}\left\langle\tilde{y}^{2}\right\rangle_{4}^{s}-\frac{1}{4}\left\langle\tilde{x}^{2}\right\rangle_{4}^{s} \\
& +\langle\tilde{x} \tilde{y}\rangle_{0}-\frac{1}{2}\langle\tilde{x} \tilde{y}\rangle_{4}^{c}+\beta_{o}^{2}\left\langle\tilde{t}^{2}\right\rangle_{2}^{s}-\beta_{o}\langle\tilde{t} \tilde{x}\rangle_{3}^{s} \\
& -\beta_{o}\langle\tilde{t} \tilde{x}\rangle_{1}^{s}-\beta_{o}\langle\tilde{t} \tilde{y}\rangle_{1}^{c}+\beta_{o}\langle\tilde{t} \tilde{y}\rangle_{3}^{c} \\
& \left(R_{o}^{2}\right)_{n}^{c}=\frac{1}{2}\left\langle\tilde{x}^{2}\right\rangle_{n}^{c}+\frac{1}{2}\left\langle\tilde{y}^{2}\right\rangle_{n}^{c}-\frac{1}{4}\left\langle\tilde{y}^{2}\right\rangle_{n-2}^{c}-\frac{1}{4}\left\langle\tilde{y}^{2}\right\rangle_{n+2}^{c} \\
& +\frac{1}{4}\left\langle\tilde{x}^{2}\right\rangle_{n-2}^{c}+\frac{1}{4}\left\langle\tilde{x}^{2}\right\rangle_{n+2}^{c}+\frac{1}{2}\langle\tilde{x} \tilde{y}\rangle_{n+2}^{s} \\
& -\frac{1}{2}\langle\tilde{x} \tilde{y}\rangle_{n-2}^{s}+\beta_{o}^{2}\left\langle\tilde{t}^{2}\right\rangle_{n}^{c}-\beta_{o}\langle\tilde{t} \tilde{x}\rangle_{n-1}^{c} \\
& -\beta_{o}\langle\tilde{t} \tilde{x}\rangle_{n+1}^{c}-\beta_{o}\langle\tilde{t} \tilde{y}\rangle_{n+1}^{s}+\beta_{o}\langle\tilde{t} \tilde{y}\rangle_{n-1}^{s} \\
& \left(R_{o}^{2}\right)_{n}^{s}=\frac{1}{2}\left\langle\tilde{x}^{2}\right\rangle_{n}^{s}+\frac{1}{2}\left\langle\tilde{y}^{2}\right\rangle_{n}^{s}-\frac{1}{4}\left\langle\tilde{y}^{2}\right\rangle_{n-2}^{s}+\frac{1}{4}\left\langle\tilde{y}^{2}\right\rangle_{n+2}^{s} \\
& +\frac{1}{4}\left\langle\tilde{x}^{2}\right\rangle_{n-2}^{s}-\frac{1}{4}\left\langle\tilde{x}^{2}\right\rangle_{n+2}^{s}+\frac{1}{2}\langle\tilde{x} \tilde{y}\rangle_{n-2}^{c} \\
& -\frac{1}{2}\langle\tilde{x} \tilde{y}\rangle_{n+2}^{c}+\beta_{o}^{2}\left\langle\tilde{t}^{2}\right\rangle_{n}^{s}-\beta_{o}\langle\tilde{t} \tilde{x}\rangle_{n-1}^{s} \\
& -\beta_{o}\langle\tilde{t} \tilde{x}\rangle_{n+1}^{s}-\beta_{o}\langle\tilde{t} \tilde{y}\rangle_{n-1}^{c}+\beta_{o}\langle\tilde{t} \tilde{y}\rangle_{n+1}^{c} \text {. }
\end{aligned}
$$

Note that from the third order onwards we have given general expressions for any order.

Analogically we derived the series for the sideward radius.

$$
\begin{aligned}
\left(R_{s}^{2}\right)_{0}= & \frac{1}{2}\left\langle\tilde{x}^{2}\right\rangle_{0}+\frac{1}{2}\left\langle\tilde{y}^{2}\right\rangle_{0}+\frac{1}{4}\left\langle\tilde{y}^{2}\right\rangle_{2}^{c}-\frac{1}{4}\left\langle\tilde{x}^{2}\right\rangle_{2}^{c} \\
& -\frac{1}{2}\langle\tilde{x} \tilde{y}\rangle_{2}^{s} \\
\left(R_{s}^{2}\right)_{1}^{c}= & \frac{1}{4}\left\langle\tilde{x}^{2}\right\rangle_{1}^{c}+\frac{3}{4}\left\langle\tilde{y}^{2}\right\rangle_{1}^{c}+\frac{1}{4}\left\langle\tilde{y}^{2}\right\rangle_{3}^{c}-\frac{1}{4}\left\langle\tilde{x}^{2}\right\rangle_{3}^{c}
\end{aligned}
$$




$$
\begin{aligned}
& -\frac{1}{2}\left(\langle\tilde{x} \tilde{y}\rangle_{1}^{s}-\langle\tilde{x} \tilde{y}\rangle_{3}^{s}\right) \\
\left(R_{s}^{2}\right)_{1}^{s}= & \frac{3}{4}\left\langle\tilde{x}^{2}\right\rangle_{1}^{s}+\frac{1}{4}\left\langle\tilde{y}^{2}\right\rangle_{1}^{s}+\frac{1}{4}\left\langle\tilde{y}^{2}\right\rangle_{3}^{s}-\frac{1}{4}\left\langle\tilde{x}^{2}\right\rangle_{3}^{s} \\
& -\frac{1}{2}\left(\langle\tilde{x} \tilde{y}\rangle_{1}^{c}-\langle\tilde{x} \tilde{y}\rangle_{3}^{c}\right) \\
\left(R_{s}^{2}\right)_{2}^{c}= & \frac{1}{2}\left\langle\tilde{x}^{2}\right\rangle_{2}^{c}+\frac{1}{2}\left\langle\tilde{y}^{2}\right\rangle_{2}^{c}+\frac{1}{2}\left\langle\tilde{y}^{2}\right\rangle_{0}+\frac{1}{4}\left\langle\tilde{y}^{2}\right\rangle_{4}^{c} \\
& -\frac{1}{2}\left\langle\tilde{x}^{2}\right\rangle_{0}-\frac{1}{4}\left\langle\tilde{x}^{2}\right\rangle_{4}^{c}-\frac{1}{2}\langle\tilde{x} \tilde{y}\rangle_{4}^{s} \\
\left(R_{s}^{2}\right)_{2}^{s}= & \frac{1}{2}\left\langle\tilde{x}^{2}\right\rangle_{2}^{s}+\frac{1}{2}\left\langle\tilde{y}^{2}\right\rangle_{2}^{s}+\frac{1}{4}\left\langle\tilde{y}^{2}\right\rangle_{4}^{s}-\frac{1}{4}\left\langle\tilde{x}^{2}\right\rangle_{4}^{s} \\
& -\langle\tilde{x} \tilde{y}\rangle_{0}+\frac{1}{2}\langle\tilde{x} \tilde{y}\rangle_{4}^{c} \\
\left(R_{s}^{2}\right)_{n}^{c}= & \frac{1}{2}\left\langle\tilde{x}^{2}\right\rangle_{n}^{c}+\frac{1}{2}\left\langle\tilde{y}^{2}\right\rangle_{n}^{c}+\frac{1}{4}\left\langle\tilde{y}^{2}\right\rangle_{n-2}^{c}+\frac{1}{4}\left\langle\tilde{y}^{2}\right\rangle_{n+2}^{c} \\
& -\frac{1}{4}\left\langle\tilde{x}^{2}\right\rangle_{n-2}^{c}-\frac{1}{4}\left\langle\tilde{x}^{2}\right\rangle_{n+2}^{c}-\frac{1}{2}\langle\tilde{x} \tilde{y}\rangle_{n+2}^{s} \\
& +\frac{1}{2}\langle\tilde{x} \tilde{y}\rangle_{n-2}^{s} \\
\left(R_{s}^{2}\right)_{n}^{s}= & \frac{1}{2}\left\langle\tilde{x}^{2}\right\rangle_{n}^{s}+\frac{1}{2}\left\langle\tilde{y}^{2}\right\rangle_{n}^{s}+\frac{1}{4}\left\langle\tilde{y}^{2}\right\rangle_{n-2}^{s}+\frac{1}{4}\left\langle\tilde{y}^{2}\right\rangle_{n+2}^{s} \\
& -\frac{1}{4}\langle\tilde{x}\rangle_{n-2}^{s}-\frac{1}{4}\left\langle\tilde{x}^{2}\right\rangle_{n+2}^{s}-\frac{1}{2}\langle\tilde{x} \tilde{y}\rangle_{n-2}^{c} \\
& +\frac{1}{2}\langle\tilde{x} \tilde{y}\rangle_{n+2}^{c} \cdot
\end{aligned}
$$

Finally, for the cross-term we obtained

$$
\begin{aligned}
\left(R_{o s}^{2}\right)_{0}= & \frac{1}{2}\langle\tilde{x} \tilde{y}\rangle_{2}^{c}+\frac{1}{4}\left\langle\tilde{y}^{2}\right\rangle_{2}^{s}-\frac{1}{4}\left\langle\tilde{x}^{2}\right\rangle_{2}^{s}+\frac{\beta_{o}}{2}\langle\tilde{x} \tilde{t}\rangle_{1}^{s} \\
& -\frac{\beta_{o}}{2}\langle\tilde{y} \tilde{t}\rangle_{1}^{c} \\
\left(R_{o s}^{2}\right)_{1}^{c}= & \frac{1}{2}\langle\tilde{x} \tilde{y}\rangle_{1}^{c}+\frac{1}{2}\langle\tilde{x} \tilde{y}\rangle_{3}^{c}+\frac{1}{4}\left\langle\tilde{y}^{2}\right\rangle_{1}^{s}+\frac{1}{4}\left\langle\tilde{y}^{2}\right\rangle_{3}^{s} \\
& -\frac{1}{4}\left\langle\tilde{x}^{2}\right\rangle_{1}^{s}-\frac{1}{4}\left\langle\tilde{x}^{2}\right\rangle_{3}^{s}+\frac{\beta_{o}}{2}\langle\tilde{x} \tilde{t}\rangle_{2}^{s}-\beta_{o}\langle\tilde{y} \tilde{t}\rangle_{0} \\
& -\frac{\beta_{o}}{2}\langle\tilde{y} \tilde{t}\rangle_{2}^{c} \\
\left(R_{o s}^{2}\right)_{1}^{s}= & -\frac{1}{2}\langle\tilde{x} \tilde{y}\rangle_{1}^{s}-\frac{1}{2}\langle\tilde{x} \tilde{y}\rangle_{3}^{s}+\frac{1}{4}\left\langle\tilde{y}^{2}\right\rangle_{1}^{c}-\frac{1}{4}\left\langle\tilde{y}^{2}\right\rangle_{3}^{c} \\
& -\frac{1}{4}\left\langle\tilde{x}^{2}\right\rangle_{1}^{c}+\frac{1}{4}\left\langle\tilde{x}^{2}\right\rangle_{3}^{c}+\beta_{o}\langle\tilde{x} \tilde{t}\rangle_{0}-\frac{\beta_{o}}{2}\langle\tilde{x} \tilde{t}\rangle_{2}^{c} \\
& -\frac{\beta_{o}}{2}\langle\tilde{y} \tilde{t}\rangle_{2}^{s} \\
\left(R_{o s}^{2}\right)_{2}^{c}= & \langle\tilde{x} \tilde{y}\rangle_{0}+\frac{1}{2}\langle\tilde{x} \tilde{y}\rangle_{4}^{c}+\frac{1}{4}\left\langle\tilde{y}^{2}\right\rangle_{4}^{s}-\frac{1}{4}\left\langle\tilde{x}^{2}\right\rangle_{4}^{s} \\
& -\frac{\beta_{o}}{2}\langle\tilde{x} \tilde{t}\rangle_{1}^{s}+\frac{\beta_{o}}{2}\langle\tilde{x} \tilde{t}\rangle_{3}^{s}-\frac{\beta_{o}}{2}\langle\tilde{y} \tilde{t}\rangle_{1}^{c} \\
& -\frac{\beta_{o}}{2}\langle\tilde{y} \tilde{t}\rangle_{3}^{c} \\
\left(R_{o s}^{2}\right)_{2}^{s}= & -\frac{1}{2}\langle\tilde{x} \tilde{y}\rangle_{4}^{s}+\frac{1}{2}\left\langle\tilde{y}^{2}\right\rangle_{0}-\frac{1}{4}\left\langle\tilde{y}^{2}\right\rangle_{4}^{c}-\frac{1}{2}\left\langle\tilde{x}^{2}\right\rangle_{0} \\
& +\frac{1}{4}\left\langle\tilde{x}{ }^{2}\right\rangle_{4}^{c}+\frac{\beta_{o}}{2}\langle\tilde{x} \tilde{t}\rangle_{1}^{c}-\frac{\beta_{o}}{2}\langle\tilde{x} \tilde{t}\rangle_{3}^{c}-\frac{\beta_{o}}{2}\langle\tilde{y} \tilde{t}\rangle_{1}^{s} \\
& -\frac{\beta_{o}}{2}\langle\tilde{y} \tilde{t}\rangle_{3}^{s}
\end{aligned}
$$

$$
\begin{aligned}
\left(R_{o s}^{2}\right)_{n}^{c}= & \frac{1}{2}\langle\tilde{x} \tilde{y}\rangle_{n-2}^{c}+\frac{1}{2}\langle\tilde{x} \tilde{y}\rangle_{n+2}^{c}+\frac{1}{4}\left\langle\tilde{y}^{2}\right\rangle_{n+2}^{s} \\
& -\frac{1}{4}\left\langle\tilde{y}^{2}\right\rangle_{n-2}^{s}-\frac{1}{4}\left\langle\tilde{x}^{2}\right\rangle_{n+2}^{s}+\frac{1}{4}\left\langle\tilde{x}^{2}\right\rangle_{n-2}^{s} \\
& +\frac{\beta_{o}}{2}\langle\tilde{x} \tilde{t}\rangle_{n+1}^{s}-\frac{\beta_{o}}{2}\langle\tilde{x} \tilde{t}\rangle_{n-1}^{s}-\frac{\beta_{o}}{2}\langle\tilde{y} \tilde{t}\rangle_{n+1}^{c} \\
& -\frac{\beta_{o}}{2}\langle\tilde{y} \tilde{t}\rangle_{n-1}^{c} \\
\left(R_{o s}^{2}\right)_{n}^{s}= & -\frac{1}{2}\langle\tilde{x} \tilde{y}\rangle_{n+2}^{s}+\frac{1}{2}\langle\tilde{x} \tilde{y}\rangle_{n-2}^{s}-\frac{1}{4}\left\langle\tilde{y}^{2}\right\rangle_{n+2}^{c} \\
& +\frac{1}{4}\left\langle\tilde{y}^{2}\right\rangle_{n-2}^{c}+\frac{1}{4}\left\langle\tilde{x}^{2}\right\rangle_{n+2}^{c}-\frac{1}{4}\left\langle\tilde{x}^{2}\right\rangle_{n-2}^{c} \\
& -\frac{\beta_{o}}{2}\langle\tilde{x} \tilde{t}\rangle_{n+1}^{c}+\frac{\beta_{o}}{2}\langle\tilde{x} \tilde{t}\rangle_{n-1}^{c}-\frac{\beta_{o}}{2}\langle\tilde{y} \tilde{t}\rangle_{n+1}^{s} \\
& -\frac{\beta_{o}}{2}\langle\tilde{y} \tilde{t}\rangle_{n-1}^{s} .
\end{aligned}
$$

\section{References}

1. B. B. Abelev et al. [ALICE Collaboration], JHEP 1506 (2015) 190 doi:10.1007/JHEP06(2015)190 arXiv:1405.4632 [nucl-ex]].

2. G. Aad et al. [ATLAS Collaboration], Phys. Rev. C 86 (2012) 014907 doi:10.1103/PhysRevC.86.014907 arXiv:1203.3087 [hep-ex]].

3. S. Chatrchyan et al. [CMS Collaboration], Phys. Rev. C 87 (2013) no.1, 014902 doi:10.1103/PhysRevC.87.014902 arXiv:1204.1409 [nucl-ex]].

4. B. I. Abelev et al. [STAR Collaboration], Phys. Rev. C 77 (2008) 054901 doi:10.1103/PhysRevC.77.054901 arXiv:0801.3466 [nucl-ex]].

5. S. S. Adler et al. [PHENIX Collaboration], Phys. Rev. Lett. $91 \quad$ (2003) 182301 doi:10.1103/PhysRevLett.91.182301 nucl-ex/0305013.

6. C. Gale, S. Jeon, B. Schenke, P. Tribedy and R. Venugopalan, Phys. Rev. Lett. 110 (2013) no.1, 012302 doi:10.1103/PhysRevLett.110.012302 arXiv:1209.6330 [nucl-th]].

7. H. Niemi, G. S. Denicol, H. Holopainen and P. Huovinen, Phys. Rev. C 87 (2013) no.5, 054901 doi:10.1103/PhysRevC.87.054901 arXiv:1212.1008 [nuclth]].

8. S. Floerchinger and U. A. Wiedemann, Phys. Rev. C 89 (2014) no.3, 034914 doi:10.1103/PhysRevC.89.034914 arXiv:1311.7613 [hep-ph]].

9. W. L. Qian, P. Mota, R. Andrade, F. Gardim, F. Grassi, Y. Hama and T. Kodama, J. Phys. G 41 (2013) 015103 doi:10.1088/0954-3899/41/1/015103 arXiv:1305.4673 [hep-ph]].

10. U. W. Heinz, J. Phys. Conf. Ser. 455, 012044 (2013)

11. M. Schulc and B. Tomášik, Phys. Rev. C 90 (2014) no.6, 064910 doi:10.1103/PhysRevC.90.064910 arXiv:1409.6116 [nucl-th]].

12. M. Nahrgang, C. Herold, S. Leupold, I. Mishustin and M. Bleicher, J. Phys. G 40 (2013) 055108 doi:10.1088/0954-3899/40/5/055108 arXiv:1105.1962 [nucl-th]].

13. Y. Tachibana, N. B. Chang and G. Y. Qin, arXiv:1701.07951 [nucl-th]. 
14. J. Crkovská et al., Phys. Rev. C 95 (2017) no.1, 014910 doi:10.1103/PhysRevC.95.014910 arXiv:1603.09621 [hep$\mathrm{ph}]$.

15. M. Luzum and P. Romatschke, Phys. Rev. Lett. 103 (2009) 262302 doi:10.1103/PhysRevLett.103.262302 arXiv:0901.4588 [nucl-th]].

16. S. Ryu, J.-F. Paquet, C. Shen, G. S. Denicol, B. Schenke, S. Jeon and C. Gale, Phys. Rev. Lett. 115 (2015) no.13, 132301 doi:10.1103/PhysRevLett.115.132301 arXiv:1502.01675 [nucl-th]].

17. G. Denicol, A. Monnai and B. Schenke, Phys. Rev. Lett. 116 (2016) no.21, 212301 doi:10.1103/PhysRevLett.116.212301 arXiv:1512.01538 [nucl-th]].

18. J. E. Bernhard, J. S. Moreland, S. A. Bass, J. Liu and U. Heinz, Phys. Rev. C 94 (2016) no.2, 024907 doi:10.1103/PhysRevC.94.024907 arXiv:1605.03954 [nuclth]].

19. B. Tomášik, Acta Phys. Polon. B 36 (2005) 2087

20. M. Csanád, B. Tomášik and T. Csörgő, Eur. Phys. J. A 37 (2008) 111

21. C. J. Plumberg, C. Shen and U. W. Heinz, Phys. Rev. C 88 (2013) 044914 Erratum: [Phys. Rev. C 88 (2013) no.6, 069901] doi:10.1103/PhysRevC.88.069901, 10.1103/PhysRevC.88.044914 arXiv:1306.1485 [nucl-th]].

22. S. Lökös, M. Csanád, T. Csörgő and B. Tomášik, Eur. Phys. J. A 52 (2016) 311

23. T. Niida [PHENIX Collaboration], Nucl. Phys. A 904-905 (2013) 439c doi:10.1016/j.nuclphysa.2013.02.043 arXiv:1304.2876 [nucl-ex]].

24. A. Adare et al. [PHENIX Collaboration], Phys. Rev. C 93 (2016) no.5, 051902 doi:10.1103/PhysRevC.93.051902 arXiv:1412.1038 [nucl-ex]].

25. J. Adams et al. [STAR Collaboration], Phys. Rev. Lett. 93 (2004) 012301

26. A. Adare et al. [PHENIX Collaboration], Phys. Rev. Lett. $\mathbf{1 1 2}$ (2014) no.22, 222301 doi:10.1103/PhysRevLett.112.222301 arXiv:1401.7680 [nucl-ex]].

27. V. Loggins [ALICE Collaboration], Nucl. Phys. A $931 \quad(2014) \quad 1088$ doi:10.1016/j.nuclphysa.2014.08.061 arXiv:1408.0068 [nucl-ex]].

28. P. J. Siemens and J. O. Rasmussen, Phys. Rev. Lett. 42 (1979) 880. doi:10.1103/PhysRevLett.42.880

29. E. Schnedermann, J. Sollfrank and U. W. Heinz, Phys. Rev. C 48 (1993) 2462 doi:10.1103/PhysRevC.48.2462 nucl-th/9307020.

30. T. Csörgő and B. Lörstad, Phys. Rev. C 54 (1996) 1390 doi:10.1103/PhysRevC.54.1390 hep-ph/9509213.

31. B. Tomášik, U. A. Wiedemann and U. W. Heinz, Heavy Ion Phys. 17 (2003) 105 doi:10.1556/APH.17.2003.1.11 nuclth/9907096.

32. F. Retiére and M. A. Lisa, Phys. Rev. C 70 (2004) 044907 doi:10.1103/PhysRevC.70.044907 nucl-th/0312024.

33. D. Teaney, Phys. Rev. C 68 (2003) 034913 doi:10.1103/PhysRevC.68.034913 nucl-th/0301099.

34. A. Jaiswal and V. Koch, arXiv:1508.05878 [nucl-th].

35. Z. Yang and R. J. Fries, J. Phys. Conf. Ser. 832 (2017) no.1, 012056 doi:10.1088/1742-6596/832/1/012056 arXiv:1612.05629 [nucl-th]].

36. U. W. Heinz, A. Hummel, M. A. Lisa and U. A. Wiedemann, Phys. Rev. C 66 (2002) 044903
37. B. Tomášik and U. A. Wiedemann, In *Hwa, R.C. (ed.) et al.: Quark gluon plasma* 715-777 hep-ph/0210250.

38. I. Melo and B. Tomášik, J. Phys. G 43 (2016) no.1, 015102 doi:10.1088/0954-3899/43/1/015102 arXiv:1502.01247 [nucl-th]].

39. J. Adams et al. [STAR Collaboration], Phys. Rev. C 71 (2005) 044906 doi:10.1103/PhysRevC.71.044906 nuclex/0411036.

40. A. Adare et al. [PHENIX Collaboration], Phys. Rev. Lett. 107 (2011) 252301 doi:10.1103/PhysRevLett.107.252301 arXiv:1105.3928 [nucl-ex]].

41. M.A. Saleh for the ALICE Collaboration and N. Tanaka for the ALICE Collaboration, talks at the XII Workshop on Particle Correlation and Femtoscopy, Amsterdam, The Netherlands, June 12-16, 2017. 